\title{
基于自相似性车载采集城市街景图像的重建
}

\author{
杨志峰1 潘慧婕 2 \\ 1 浙江省测绘科学技术研究院 2 浙江省商务人力资源交流服务中心 \\ DOI:10.32629/gmsm.v3i4.827
}

\begin{abstract}
[摘 要] 普通的车载系统为了保证图像实时、高速的显示以及便于图像的存储, 所以捕获的图像分别率 会比较低。这种方式会导致无法在突发情况下从图像中提取有效信息。针对这种情况, 本文就如何对低 分辨率图像进行超分辨率重建进行分析研究。本文研究采用的算法是基于图像的自相似性,通过放射变 换和透视变换的方式匹配图像块,并在过程中对图像信息采用高频补偿的方式找回丢失的图像信息, 不 断丰富图像的细节信息, 使图像达到超分辨率的重建。此次试验通过此算法并与三种典型的算法进行比 对,比对结果表明该算法在评价指标上要强于其他算法,不仅提高了图像的分辨率还同时保持了图像的 细节信息。
\end{abstract}

[关键词] 仿射变换; 透视变换; 高频补偿方式

中图分类号：P237 文献标识码：A

\section{1 算法的总体概述}

如图1 (a) 所示, 传统方法是基于单个 图像的自相似性来重建图像的过程, 从图 中可以看出, 低分辨率图像 $\mathrm{I}_{0}$ 中给出的每 一个的图像块在采样图像 $\mathrm{I}_{-1}$ 中都能找到 相对应的图像块, 这个图像块在低分辨率 图像 $\mathrm{I}_{0}$ 中的对应位置是作为高分辨率图 像 $\mathrm{I}_{1}$ 中的预测出现的。所以高分辨率图像 $I_{1}$ 是复制粘贴了这个图像块的。

基于图像自相似性的特性, 在原有 的仿射变换算法上增加透视变换的算法, 增加对相似图像搜索。此综合算法可以 有效的提高图像块匹配的精确度以及图 像的清晰度。如图 1 (b) 所示。通过图中
所示可以看出, 在 $I_{0}$ 中的每一个目标源 图像块 $\mathrm{P}$, 我们都能在下面的采样图像 $\mathrm{I}_{-1}$ 中找到与它像对应的源图像块 $\mathrm{Q}$ 。他 们的变换参数是对相似区域通过对比低 分辨率的图像和采样图像来获得的, 如 图所示, 提取源图像块在 $\mathrm{I}_{0}$ 中的高分辨 率形式 $Q_{H}$ 。对 $Q_{H}$ 的求逆变换可以获得 $P H$, 在低分辨率图像中P的对应位置粘贴图 像块 $\mathrm{P}_{\mathrm{H}}$, 用以对图像进行重建。由此总结 出此算法是在仿射变换和透视变换的过 程中对图像信息进行高频补偿, 最终获 得超分辨率的图像。此算法结合不同的 方式从多方面的角度入手来对图像进行 重建。

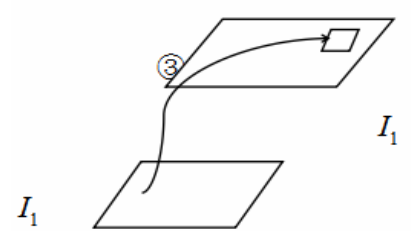

$I_{1}$

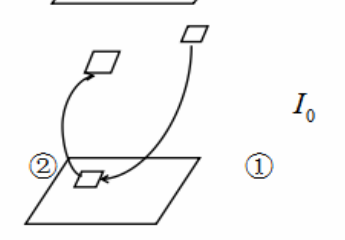

$I_{-1}$

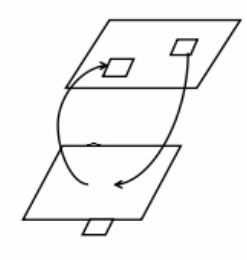

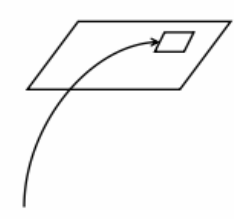
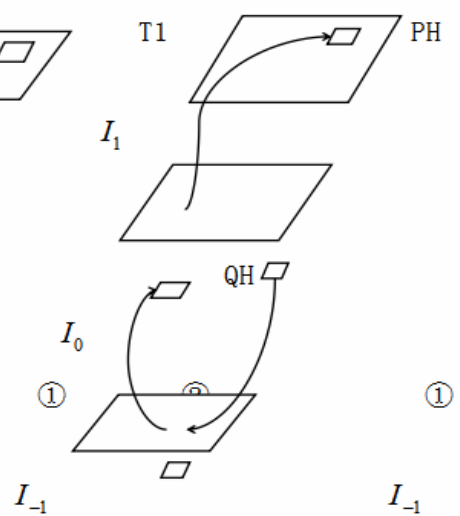

(b)本文方法

\section{2 检测图像平面确定平面参数}

2.1 检测定位平面

首先我们需要对低分辨率的图像的 三维结构进行定位, 以便于来获得透视 变换矩阵。本文采用的检测方法是通过 选取消失点并找到对应的平面, 对消失 点进行分组, 分析各项数据的方式最后 对平面进行识别。首先我们需要随机选 取三个消失点, 并提取图像的直线段, 找 到消失点对应的三个平面。

2. 2 平面参数的计算

本文采用检测消失点方式, 对图像 的平面参数进行计算。如图 2 , 定位 3 个平 面, $\mathrm{m}_{\mathrm{i}}$ 表示索引不同的平面, 变换矩阵 $\mathrm{T}_{\mathrm{i}}$ 的参数为 $\theta_{i}=\left(s_{i}, m_{i}\right)$, 其中

$$
s_{i}=\left(s_{i}^{x}, S_{i}^{y}, S_{i}^{s}, S_{i}^{\theta}, S_{i}^{\partial}, s_{i}^{\beta}\right)
$$

为六个维度的仿射变换参数。定义 变换矩阵为:

$$
\mathrm{T}_{i}\left(\theta_{i}\right)=\mathrm{H}\left(t_{i}, s_{i}^{x}, s_{i}^{y}, m_{i}\right) S
$$

$$
\left(s_{i}{ }^{x}, s_{i}{ }^{y}\right) \mathrm{A}\left(s_{i}^{\alpha}, s_{i}^{\beta}\right)
$$

$\mathrm{H}$ 表示获得的目标图像块与源图像 块位置的透视变换以及平面参数。 


$$
\begin{aligned}
& S\left(s_{i}{ }^{s}, s_{i}{ }^{\theta}\right)=\left[\begin{array}{cc}
s_{i}^{s} R\left(s_{i}^{\theta}\right) & 0 \\
0^{\mathrm{T}} & 1
\end{array}\right](2) \\
& \mathrm{A}\left({s_{i}}^{\alpha}, s_{i}^{\beta}\right)=\left[\begin{array}{ccc}
1 & s_{i}^{\alpha} & 0 \\
s_{i}^{\beta} & 1 & 0 \\
0 & 0 & 1
\end{array}\right]
\end{aligned}
$$

\section{3 最近邻域估计}

为了在图像块中搜索、估计最近邻 域, 我们通过增加透视变化算法的方式 得到变换矩阵 $\mathrm{T}_{\mathrm{i}}\left(\theta_{\mathrm{i}}\right)$ 的最优解。相比之 前的二维空间域的变化, 此次算法采用 对三维空间搜索的方式, 估计每一个平 面的最近邻域。对每个平面空间的最近 邻域的目标函数进行定义, 我们用公式 (5表示), 在公式中参数 $\theta_{i}$ 是构建变换 矩阵 $\mathrm{T}_{\mathrm{i}}\left(\theta_{\mathrm{i}}\right)$ 的未知参数:

$$
\begin{aligned}
& \min _{\left\{\theta_{i}\right\}} \sum_{i \in \Omega} E_{\text {app }}\left(t_{i}, \theta_{i}\right)+E_{\text {plane }} \\
& \left(t_{i}, \theta_{i}\right)+E_{\text {scale }}\left(t_{i}, \theta_{i}\right)
\end{aligned}
$$

\section{1 表面纹理代价函数}

对源图像块与目标图像块的相似性 的比较, 我们用表面纹理代价函数表示。

$W_{i}$ 是方差为三的高斯加权矩阵:

$$
\mathrm{E}_{\text {app }}\left(t_{i}, \theta_{i}\right)=\left\|W_{i}\left(P\left(t_{i}\right)-Q\left(t_{i}, \theta_{i}\right)\right)\right\|_{2}^{2}
$$

\section{2 平面兼容的代价函数}

本文算法使用消失点检测, 可以有 效地定位平面。通过定位得到的三维平 面可以用于引导图像块的搜索空间。 $\operatorname{Pr}\left[m_{i} l(x, y)\right]$ 为像素位置 $(\mathrm{x}, \mathrm{y})$ 的平 面 $\mathrm{mi}$ 的后验概率:

$$
\begin{gathered}
E_{\text {plane }}=-\lambda_{\text {plane }} L n \\
\left(\operatorname{Pr}\left[m_{i} \mid\left(s_{i}^{x}, s_{i}^{y}\right)\right] \times \operatorname{Pr}\left[m_{i} \mid\left(t_{i}^{x}, t_{i}^{y}\right)\right]\right)
\end{gathered}
$$

3. 3 缩放的代价函数

对源图像块与目标图像块的最近邻 域进行比较, 比较的结果可能出现零解, 为了避免零解出现, 我们引入缩放的代 价函数。在函数中我们用SRF表示超分辨

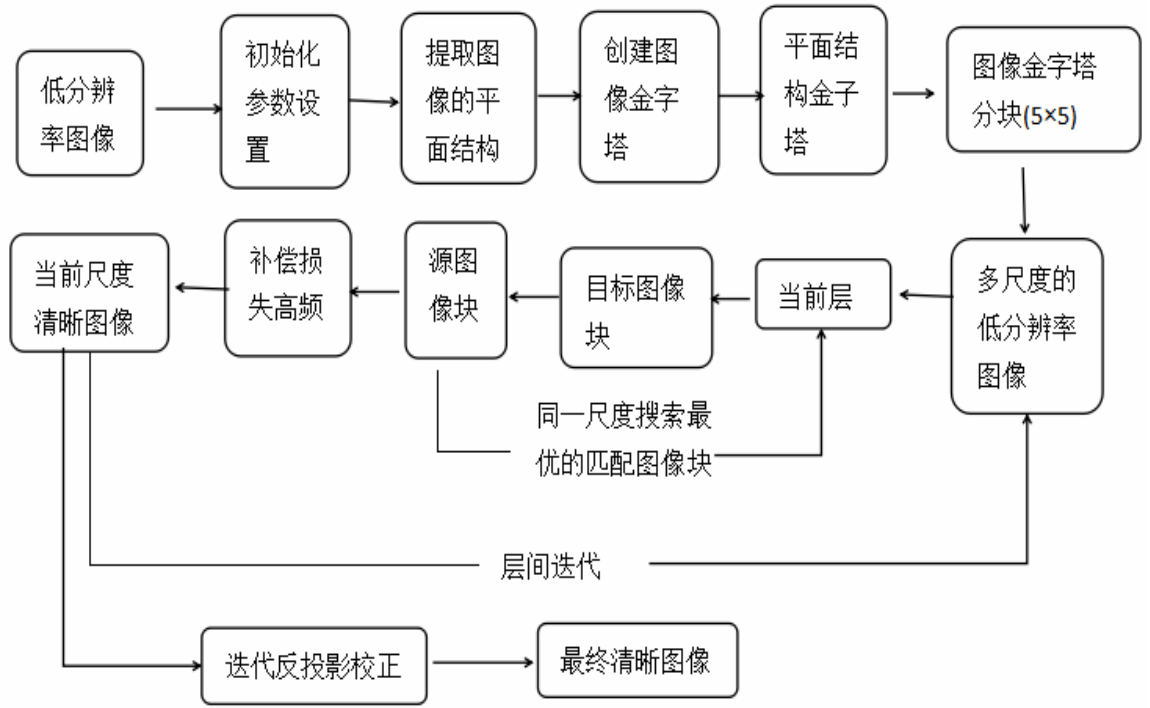

图 2 算法的总体框图

率重建因子, Scale $\left(\mathrm{T}_{\mathrm{i}}\right)$ 表示投影变换矩 阵的尺度估计：

$E_{\text {scale }}=\lambda_{\text {scale }} \min \left(0, \operatorname{SRF}-\operatorname{Scale}\left(\mathrm{T}_{i}\right)\right)$

对于源图像块采样的缩放尺度的评 估我们使用 $\mathrm{T}_{\mathrm{i}}$ 表示。公式如下:

$$
\operatorname{Scale}\left(\mathrm{T}_{i}\right)=
$$

$\sqrt{\operatorname{det}\left[\begin{array}{cc}\mathrm{T}_{1,1}-\mathrm{T}_{1,3} \mathrm{~T}_{3,1} & \mathrm{~T}_{1,2}-\mathrm{T}_{1,3} \mathrm{~T}_{3,2} \\ \mathrm{~T}_{2,1}-\mathrm{T}_{2,3} \mathrm{~T}_{3,1} & \mathrm{~T}_{3,1}-\mathrm{T}_{2,3} \mathrm{~T}_{3,2}\end{array}\right]}$

\section{4 图像块的高频补偿}

本文采用仿射变换和透视变换, 增 加图像的搜索范围, 总结出此算法是在 低分辨率的图像的重建过程中对每个图 像块进行高频补偿, 补偿每个图像块的 详细信息, 以此来实现超分辨率图像的 重建过程。其算法的总体框图如图。

\section{5 实验结果及分析}

5. 1 实验对比结果

本文采用仿射变换和透视变换并补 偿每个图像块丢失的高频信息, 最终重 建图像的过程, 此次对算法的仿真使用 的是Matlab R2017a软件。

我们选取 6 张车载中分辨率为的 $92 * 256$ 像素的城市街景图像进行实验对 比与验证。我们对图像分别进行不同倍
数的放大, 通过不同的放大倍数中不同 场景的两幅图像进行对比, 来验证不同 算法对图像重建的质量与效果。我们通 过局部放大的方式来更清楚的比较和观 察图像的细节, 展示图像不同的特征区 域。在将城市街景图像放到2、3、4倍时 可以看出, 本算法对于低分辨率图像的 重建效果和质量更好, 对于图像的细节 信息比其他算法更加清晰。

5. 2 图像质量盲评估

上面的比对方式是从人的视觉上来 看, 本文采用的重建算法效果更好, 图像 更清晰。由于人的视觉有一定的偏差与 极限, 我们也需要通过客观的数据指标 来对重建后的图像质量进行对比。由于 我们无法获取原始的高分辨率参考图像, 所以我们采取无参考图像的质量评价方 法, 也就是盲评估的方式进行数据对比, 将采用不同算法的重建图像的各项数据 进行比对, 从而得出哪种算法得到的图 像质量和效果更好。

我们通过图像信息熵、图像对比度 以及算法复杂度等信息来比较不同算法 得到的重建图像质量, 对比各种数据, 其 中图像携带的信息量用信息熵表示, 图 像的质量优劣是由图像携带信息量的多 少来决定的, 所以信息熵数值越大, 图像 的质量越好; 图像的边缘强度可以反映 图像纹理的清晰度, 图像边缘强度的值 越大表明该图像的纹理越清晰; 盲图像 


\title{
航测遥感内业数据处理关键技术探究
}

\author{
刘欣 \\ 青海省基础测绘院 \\ DOI:10.32629/gmsm.v3i4.771
}

\begin{abstract}
[摘 要] 航测遥感工作中数据处理是非常关键的一项技术, 直接影响到航测遥感的结果。随着科学技术 的进步, 航测遥感内业数据处理技术水平正在不断提高。随之而来的, 在航测遥感数据处理方面也取得了 很大的成就, 这也就促进了整个航测工作的开展。基于此, 文章主要探讨航测遥感内业数据处理关键技术 的研究与应用。
\end{abstract}

[关键词] 空间数据; 航测遥感; 数据处理; 关键技术

中图分类号：P237 文献标识码：A

\section{1 空间数据的概述}

\section{1数字线划图}

该模式关键是对地图中的相关基础 信息进行采集, 由于具备实际数据生产 能力而且可以与空间分析要求相满足, 数字线划图能够采用Geoway3.5软件, 基 于 $1: 10000$ 的搜啊秒图像, 采集与编辑图 形数据。

\section{2 数字正射影像图模式}

该模式主要是对扫描完成后的遥感 影像与数字化航空照片展开处理, 从而 形成有用数据, 数字正射影像图模式的 主要特点在于信息量丰富、精确度高、 真实性强等, 能够将其当作背景将地图 中的新信息提取出来。

质量评价指标的值在 0 到 100 之间, 该值 的大小直接说明图像的质量的优劣, 该 值越大, 重建的图像的质量越好。由表中 的数据可知, 从总体上来讲本文的算法 优于其他算法。本文采用仿射变换和透 视变换算法, 对每个图像块的高频信息 进行补偿, 最终实现图像的重建。综合不 同算法重建图像的各种数据以及各方面 的评价指标, 可以知道, 本文使用的算法 比其他算法在图像分辨率和执行效率上 有更高的提升。

\section{6 结语}

在有突发状况需要分析取证更多 的图像信息时, 重建车载图像能够实现

\section{3数字栅格地图}

数字栅格地图模式与地形图的一致 性较高, 在颜色、规格以及形状等方面能 够真实的模拟实际生活中的地形, 具有 很大的优点。数字栅格地图模式可以通 过距离与密度进行绘制, 帮助工作人员 更好的进行全数字测量空间数据分析。

1. 4数字高程模式

数字高程模式在进行空间数据测量 的时候可以控制地表形态, 该模式是在 高斯平面上不规则或者规则点的平面坐 标以及高度的数据集子, 可以配套提供 离散高程点数据。例如大比例尺的地形 图, 虽然它的综合程度较低, 但是它能 够清晰地表现出地形地貌, 但是小比例

什么样的的质量和效果至关重要, 为了 我们在需要时可以获取更多的图像细 节, 本文对不同算法能够达到对低分辨 率街景图像重建的质量和效果进行了 比对和研究。本文基于图像的自相似性, 通过仿射变换和透视变换, 在过程中对 每个图像丢失的信息进行高频补偿, 对 低分辨率的图像由低到高进行重建, 不 断丰富图像的细节信息, 使图像达到超 分辨率的重建。通过各种视觉观察、数 据以及指标的对比, 本文采用的算法有 效的对图像进行了重建, 并对图像的质 量有一定的保证。图像没有模糊化, 也 没有过度锐化。图像的质量和清晰度有
尺的地形图, 只能表现出地形地貌的大 体轮廓, 无法清晰真实的展现出其地形 地貌。

\section{2 航测遥感技术的概念}

航测, 也叫作摄影测量与遥感, 是测 绘学中遥感技术的一种。遥感技术需要 融合计算机、电子、空间等众多的科学 技术, 属于一种新型的技术。航测遥感技 术能够通过非接触的方式来取得想要测 量目标的时间与空间信息。应用这种技 术不但可以对常规的目标进行定位, 还 可以通过应用外层空间传感器获得目标 的影像信息与非影像信息, 为人们对自 然界各种事物的研究提供更加全面的数 据。与此同时, 航测遥感技术在国家的国

明显的提高。但是该算法在执行效率上 还有待提升。

\section{[参考文献]}

[1]李键红, 吴亚榕, 吕巨建. 基于 自相似性与多任务高斯过程回归的 单帧图像超分辨率重建 [J]. 光学精密工 程,2018,26(11):205-217.

[2]向文,张灵,陈云华,等.基于结构自 相似性和形变块特征的单幅图像超分辨率 算法[J].计算机应用,2019,39(01):281-286.

[3]杨伟,谢维成,蒋文波,等.基于自 相似性车载采集城市街景图像的重建 [J].计算机应用,2017,37(03):817-822. 